\title{
Direct retrieval from elaborated memory traces
}

\author{
NEFF WALKER \\ American University of Beirut, Beirut, Lebanon
}

\begin{abstract}
Four experiments were performed to investigate the beneficial effects of elaborative processing on recall performance. Specifically, the experiments investigated J. R. Anderson's (1983) claim that increasing the number of associative pathways between propositions in a memory trace improves recall performance through direct retrieval. In this study, procedures were used that allowed the amount of elaboration to be systematically varied while the use of inferential reconstruction to aid recall was eliminated, thus providing a test of J. R. Anderson's model. Across all experiments, the results showed that recall of a name was better when it had been stored in a less elaborated memory trace. The results suggest that when reconstructive processes are eliminated, elaboration decreases rather than increases recall performance.
\end{abstract}

Much recent research has focused on the beneficial effects of elaborative processing on recall performance (J. R. Anderson, 1983). The concept of elaboration has largely grown out of and replaced the earlier concept, depth of processing, to become the major theoretical explanation for differences in recall performance. J. R. Anderson and his colleagues (e.g., J. R. Anderson, 1983; J. R. Anderson \& Reder, 1979; Bradshaw \& J. R. Anderson, 1982) have been at the forefront of the effort to clearly delineate the ways in which elaborative processing occurs and affects recall performance. This work has proven fruitful, and numerous researchers have focused on elaboration as a central process in memory (e.g., Eysenck, 1979; Stein, Morris, \& Bransford, 1978). The current theoretical model that most clearly delineates the ways in which elaborative processing affects recall is that of J. R. Anderson (1983). The four experiments reported in this paper were designed to evaluate some of the suppositions of this theoretical model.

According to J. R. Anderson (1983), elaborative processing works in three ways to increase recall performance. The first and most important way is through inferential reconstruction. This is generally the same concept that was called inferential redundancy by $\mathbf{J}$. R. Anderson in his earlier work (Bradshaw \& J. R. Anderson, 1982), and has been suggested to increase recall performance in the following manner: If a person has used elaborative processing at the time of encoding, the memory trace contains not only the given information, but also other related propositions. A cue given to retrieve a proposition from the trace may not serve to activate the to-be-recalled proposition to a degree that allows for retrieval. However, the activation that spreads from the cue may serve to activate one or more propositions from

The author is grateful for the support received from the University of New Hampshire during the period of data collection for this study. Thanks are also due to Jennifer Bryce, John Limber, and the reviewers of this paper for their helpful comments. Reprint requests may be sent to the author at American University of Beirut, 850 Third Avenue, New York, NY 10022. the elaboration. This information, once retrieved, can then be used to infer the schema that was used at the time of encoding. The schema can then be used to embellish on the recalled elaborations, and through this process, the to-be-recalled proposition may be generated. The effectiveness of this process, of course, depends on the degree of overlap in meaning between the elaboration, the schema, and the to-be-recalled proposition. This is basically the same process that other researchers (e.g., Stein et al., 1978) have proposed as the major reason for the beneficial effect of elaborative processing on recall performance.

The other two mechanisms that J. R. Anderson's model (1983) proposes are both direct ways that elaborative processing increases recall performance. By direct, it is meant that activation can spread down existing links between the proposition used as a cue and the to-be-recalled proposition. Therefore, recall performance is not dependent upon one's ability to generate or infer the to-berecalled proposition. The first of the two ways that J. R. Anderson suggests that elaboration can serve to increase recall performance is by providing alternate sources of activation. As in the case of inferential reconstruction, one may assume that there are some cases in which there is enough activation to retrieve some propositions from the elaboration when the target proposition cannot be retrieved. Once these propositions have been retrieved, they serve as a new source of activation. This is similar to what occurs in inferential reconstruction, except that inferential reconstruction does not rely upon one's ability to generate the target proposition. Rather, it assumes that a pathway will still exist between the retrieved proposition and the target proposition and that when the elaboration is focused upon it, it will serve to spread more activation toward the target proposition.

According to J. R. Anderson (1983), the final and least important way that elaborative processing serves to increase recall performance is through the redirection of activation. According to J. R. Anderson's model, when one item from the memory trace is given as a cue, it serves 
to spread activation not only to the other propositions in the trace but also to any prior associates of the cue proposition that had previously been stored in memory. This means that the amount of activation that spreads to the to-be-recalled proposition depends on the proportion of the overall number of pathways in the trace that lead to the to-be-recalled proposition. For example, if one assumes (for simplicity) equal strength of pathways to all propositions, a cue that has five prior associates and only one connection to the to-be-recalled proposition will send to the to-be-recalled proposition only one sixth of the activation spreading out from it. If, however, elaborative processing during encoding resulted in additional propositions that serve to connect the cue and the target proposition, the amount or proportion of the total activation energy that is directed toward the target proposition is increased. Although the amount of activation is not directly proportional to the number of pathways because of the loss of energy as the activation spreads indirectly between cue and target propositions, the overall amount of activation can be increased. Elaborative processing serves to redirect the activation away from prior associates and toward the target proposition; therefore, the probability of recall increases.

An important point to note about J. R. Anderson's (1983) formulation is the importance of the structure of the memory trace that results from the elaborative processing. For elaboration to result in redirection of activation or the provision of additional sources of activation, the memory trace must have a particular structure. This structure must include a high degree of interrelatedness between the propositions in the elaboration and the original material. This interrelatedness is necessary if the alternative pathways from the cue to the target proposition are to exist. The addition of random bits of information to a trace may or may not provide the appropriate interrelatedness. J. R. Anderson addresses this distinction by distinguishing between relevant and irrelevant fan. J. R. Anderson refers to a relevant fan as an elaboration that results in extra propositions that provide additional pathways between propositions in the original information. An irrelevant fan, on the other hand, refers to a situation in which elaborative processing results in additional propositions that have pathways leading away from the original information. Thus, according to J. R. Anderson's (1983) model, when elaborative processing results in a memory trace that has a high degree of interrelatedness between the propositions, recall performance is enhanced.

In support of the concept of inferential reconstruction, J. R. Anderson (1983) relies heavily on an earlier study by Bradshaw and J. R. Anderson (1982). This study compared the recall of specific information presented in three different contexts. The first context, called the singlesentence context, presented a single fact (the target sentence) about a famous person. The second context, called the unrelated context, presented the target sentence in addition to two other sentences containing pieces of information about that person. However, these latter two pieces of information were not specifically related to the information given in the single target sentence, except that all sentences contained information about the same person. The third context, the related context, again presented the target sentence about the person, but also provided two sentences that allowed the reader to infer the specific information presented in the target sentence. The additional sentences either provided the cause or stated the consequences of the information contained in the target sentence. Thus, Bradshaw and J. R. Anderson were testing whether inferential reconstruction (the related condition) would increase recall of the central target fact. Using the names of the historical figures as cues, Bradshaw and J. R. Anderson obtained recall data that supported their hypothesis. The related condition produced the highest level of recall for the target sentence. The unrelatedsentence condition produced the lowest level of recall performance, with the single-sentence condition falling in between. Although these results certainly offer strong support for J. R. Anderson's (1983) position concerning the facilitative effect of inferential reconstruction on recall performance, the study was not designed to, and does not, provide any evidence showing that Anderson's other two proposed mechanisms, redirecting activation or providing additional sources of activation, also play a role in improving recall performance.

Although J. R. Anderson (1983) was able to provide strong evidence for the role of inferential reconstruction, the evidence he provided for the other two proposed mechanisms is less clear. When discussing the concept of elaborative sources of activation, J. R. Anderson referred to earlier work on contextual cues (J. R. Anderson \& Bower, 1972) and configurally related sentence cues (R. C. Anderson \& Ortony, 1975). Both types of cues have been used to show that words that relate to concepts contained in a sentence serve as better cues for the sentence (or a proposition from the sentence) than do some words actually drawn from the sentence. For example, R. C. Anderson and Ortony found that the word actress, when used as a cue, promoted higher recall for the sentence "Nurses are often beautiful" than it did for the sentences "Nurses have to be licensed" and "Landscapes are often beautiful." 'This was interpreted as evidence that actress served to activate the concept of glamour. This concept, once activated, spread activation through its prior association to nurse and beautiful in the first case, but only to one word (nurse in the second example and beautiful in the last example) for the other two sentences (J. R. Anderson, 1983). This increase in the amount of activation, then, could explain the difference in recall performance. However, the results of this study could also be adequately explained through the concept of inferential redundancy. If a person initially stores with the sentence a general schema or macroproposition as part of the elaboration (as suggested by numerous researchers, e.g., van Dijk \& Kintsch, 1983), one could see how inferential reconstruction could also explain these results. In the first example given above, a general theme might be "beautiful 
women." In the next two examples, the themes might be "professional licensing" and "gardening." These are not the only themes that could apply to the sentences, obviously, but they do seem to be possible themes for each sentence. If these themes were stored, the cue word $a c$ tress would have a much better chance of activating the schema or theme for the first example than for the latter two examples. Once this activation occurs, the recall difference would then be explained in the same way as the results of Bradshaw and J. R. Anderson (1982), through the process of inferential reconstruction. Inferential reconstruction could work in this case by the cue word's activating a general schema or macroproposition for glamour or glamorous women. This schema would activate subsets or exemplars of the schema (e.g., stewardesses, actresses, nurses), and the subject would then try to recognize the correct item. Thus, results such as those reported by R. C. Anderson and Ortony could be explained without reliance upon increased amounts of activation directly reaching the target proposition.

This is not to say that J. R. Anderson's (1983) formulation of how elaborative processing increases recall performance is incorrect. It does suggest, however, that his claims for the role of redirecting activation and providing alternative sources of activation have not been adequately tested. The data that J. R. Anderson used to support the latter two mechanisms can be explained in terms of inferential processes alone. In fact, data exist that somewhat support this position. Yekovich and Manelis (1980) compared the recall of short paragraphs that were either high or low on coherence (relevant or irrelevant fan) across amounts of delay and types of recall conditions. They found that in both the free and cued immediate recall conditions, the high-coherence passages were recalled better than were the low-coherence passages. Subjects in the delayed free recall condition, however, evidenced slightly higher retention of the low-coherence passages. In interpreting their results, Yekovich and Manelis suggested that under conditions in which reconstructive processes play a small role in determining performance, other factors, such as search processes, may play a large role in retention performance.

In addition to this, a line of research pursued by Stein, Bransford, and their colleagues (e.g., Stein, Littlefield, Bransford, \& Persampieri, 1984; Stein et al., 1978) focused on the constraints on effective elaboration. In interpreting their findings, Stein et al. (1984) suggested that elaborations that provide semantically congruous information do not improve recall performance unless the elaboration strengthens the thematic relationship between the concepts. Their formulation strongly suggests that providing alternative sources of activation or redirecting activation may not be mechanisms by which elaborative processing increases recall performance.

The purpose of the experiments reported in this paper is to investigate the relative effects of the processes that J. R. Anderson's (1983) theoretical model posits as underlying the beneficial effects of elaboration. Specifically, the study was designed to provide a test of the relative effects of elaborative processing on recall through direct retrieval. Four experiments were designed in which the relative effects of inferential reconstruction were isolated from the effects of redirecting activation and the provision of additional sources of activation.

In trying to isolate the various ways in which elaborative processing may affect recall, one must devise a test that maintains the relevant fan of the memory trace while eliminating the effects of inferential reconstruction. To do this, I designed four experiments that used materials similar to those of Bradshaw and J. R. Anderson (1982). In their study, a central fact was presented in three conditions that varied in the amount of inferential redundancy contained in the memory trace. However, the memory trace of the three conditions also differed in terms of the amount of network redundancy that existed among the traces. If one compares the memory traces for a central fact (see Table 1 for examples), one notes that there are differences between the single and the related conditions in the number of connections between the name and the facts associated with the figure. In the single condition, there is one pathway connecting the name to the single fact. In the related condition, the name is connected to three different facts. Therefore, according to this model of elaboration (J. R. Anderson, 1983), the name of the historical figure is stored in a more elaborated trace in the related condition. Recall of the name is more likely, the theory suggests, when the person is presented in the related condition than in the single condition. Finally, one expects the recall of the name in the unrelated condition to be lower than in the related condition or in the single condition, as the additional facts result in an irrelevant fan effect in which activation is directed away from the to-be-recalled name. However, if the two proposed mechanisms (J. R. Anderson, 1983) do not serve to increase recall performance, one expects recall of a name in the single-fact condition to be higher than recall of the name in either the related- or unrelated-fact conditions.

In the experiments presented in this paper, subjects were asked to memorize information about historical figures.

Table 1

Sample Stimuli

Single Condition

Lavoisier helped collect taxes to support his research.

Related Condition

Lavoisier helped collect taxes to support his research.

This fact resulted in:

Lavoisier was considered a strong supporter of the monarchy.

Lavoisier was beheaded during the French Revolution.

Unrelated Condition

Lavoisier helped collect taxes to support his research.

This fact is unrelated to:

Lavoisier believed that exact measurements were essential to the study of chemistry.

Lavoisier originally was interested in studying geology. 
As in the Bradshaw and J. R. Anderson (1982) study, the information was presented in a single-fact, related-fact, or unrelated-fact condition. However, unlike subjects in their study, the subjects were provided with the facts and asked to recall the historical figures. This task differs from that of Bradshaw and J. R. Anderson in that subjects are not able to reconstruct the names given the facts as cues. Therefore, this task seems to provide an ideal test of the effectiveness of elaborative processing in increasing recall performance through direct retrieval.

\section{EXPERIMENT 1}

\section{Method}

Subjects. The subjects were 45 undergraduate students at the University of New Hampshire. The students received course credit for their participation.

Materials. The materials for the experiment were a set of biographical facts about 21 people. The facts were constructed to be similar to those used by Bradshaw and J. R. Anderson (1982). For each of the 21 historical figures, 5 facts were chosen. Facts were selected from biographies about each person, and pilot data indicated that there was little probability that the facts would be known by subjects in the experiments.

The 5 facts were used to create three different fact conditions for each historical figure. First, there was 1 central fact for each figure. The remaining 4 facts were of two types: Two of the facts were unrelated to the central fact, and 2 of the facts were directly related to the central fact (related facts) and were either probable results or probable causes of the central fact. For the 21 central facts, 10 had caused-by related facts and 11 had resulted-in related facts.

Subjects read all 21 central facts. The historical figures were presented in one of the three fact conditions: (1) single: central fact alone; (2) related; central fact plus 2 related facts; or (3) unrelated: central fact plus 2 unrelated facts. Each subject read a set of facts about seven historical figures in each of the three fact conditions. Placement of a historical figure in a condition was counterbalanced so that each figure was presented in each condition to an equal number of subjects. Presentation order of the historical figures was randomly determined and held constant for all subjects.

Procedure. Subjects participated in one 45-min experimental session. All subjects were randomly assigned to one of three groups, and each group was run separately. Subject groupings were based upon the placement of the historical figures in a particular experimental condition. Each subject was given a booklet that contained the passages about the historical figures. One historical figure and its associated fact(s) were presented on each page. The central fact plus the name was always presented at the top of the page, with the additional facts listed directly below, as shown in Table 1. Subjects were instructed to read and memorize the information about each historical figure and told that they would later be asked to remember the information. It was stressed that both the names and the associated facts needed to be remembered in order to perform well on the later test. Memorization of the facts was performed during two trials. First, the subjects were paced through the booklet, with $30 \mathrm{sec}$ for each item in the related-and unrelated-fact conditions and $15 \mathrm{sec}$ for the items in the single-fact condition. Subjects were cued to turn the page by the sounding of a tone. Following the first trial, subjects were given a 1-min break before the second trial. On the second trial, subjects were given only $10 \mathrm{sec}$ to review each of the 21 items, regardless of condition.

Following the second memorization trial, all subjects spent $4 \mathrm{~min}$ working on a 10-item number-series task. At the conclusion of the distractor task, subjects were given a second booklet that contained the 21 central facts, but with the names of the historical figures missing. Subjects were given $15 \mathrm{~min}$ to write down the name of the historical figure associated with each fact.
In addition to the main experiment, a preliminary control experiment was performed. The purpose of the pilot procedure was to ensure that the procedures and stimuli used were analogous to those used by Bradshaw and J. R. Anderson (1982). Of primary importance was the need to show that there was a difference in the degree of relationship between the central fact and the sets of related and unrelated facts. In the pilot portion of the study, all procedures were as described earlier, except that 24 additional subjects were provided with the 21 names and asked to recall the facts. If the two sets of facts did vary in terms of how related they were to the central fact, central-fact recall should be better in the related- than in the unrelated-fact condition.

\section{Results and Discussion}

Initially a one-way analysis of variance was performed on the number of correctly recalled (paraphrased) central facts in the pilot study. This analysis yielded a significant effect of fact condition $(F=8.20, p<.001)$. A Tukey comparison revealed that central facts were recalled more in the related-fact (mean $=68.3 \%$ ) and single-fact conditions (mean $=65.0 \%$ ) than in the unrelated-fact condition (mean $=45.0 \%$ ). This pattern of results is similar to that found in the experiments performed by Bradshaw and J. R. Anderson (1982). Although the difference in recall performance was not significant between the related- and the single-fact conditions, the results did parallel those of Bradshaw and J. R. Anderson (1982). In the three experiments reported by them, only in the experiment in which there was a 1-week delay between learning and retrieval was the difference between these two conditions significant. In the other two experiments, in which the procedures more closely resembled those used in this study, the pattern of results was identical (in terms of significant differences and ordering of the means) to those found here. Therefore, it seems that the related and unrelated facts did differ in terms of their relationship to the central fact.

Table 2 shows the mean percentage of correctly recalled names by condition in Experiment 1. A one-way analysis of variance on the number of correctly recalled names revealed a significant effect for condition $(F=11.08$, $M S \mathrm{e}=1.27, p<.001)$. A Tukey comparison revealed that the single-fact condition led to higher recall than did the related-and unrelated-fact conditions, whereas recall in the related-and unrelated-fact conditions did not differ significantly.

A check was made on the type of errors made on the recall test. Errors were of three types: (1) omissionsproviding no answer; (2) confusions-giving a historical

Table 2

Mean Number (and Mean Percentage) of Correctly Recalled Names by Condition

\begin{tabular}{cccc}
\hline & \multicolumn{3}{c}{ Condition } \\
\cline { 2 - 4 } Test Type & Single & Related & Unrelated \\
\hline & Experiment 1 \\
Central-Fact Cue & $3.54(50.6 \%)$ & $2.80(40.0 \%)$ & $2.40(34.3 \%)$ \\
& Experiment 2 & \\
Central-Fact Cue & $3.89(55.6 \%)$ & $3.08(44.0 \%)$ & $3.02(43.2 \%)$ \\
Multiple-Fact Cue & $4.31(61.5 \%)$ & $3.77(53.8 \%)$ & $3.65(52.2 \%)$ \\
\hline
\end{tabular}


figure from the stimuli, but placing it with the wrong central fact; and (3) intrusions-providing a name not included in the stimuli. All errors were scored, and it was found that over the three conditions, $92.2 \%$ of the errors were omissions and, with the exception of one intrusion, all other errors were confusions. An analysis of error type by fact condition revealed no difference due to fact condition.

This pattern of results is clearly not supportive of the prediction drawn from J. R. Anderson's (1983) theoretical model. Name recall was better when the name was stored in a less elaborated memory episode. This strongly suggests that processes that rely upon direct retrieval do not play an important role in explaining the generally beneficial effect that elaborative processing has upon recall.

One possible problem with the results of Experiment 1 is that the differences obtained could be interpreted in terms of a cue-overload effect. According to the cueoverload principle (Mueller \& M. J. Watkins, 1977; M. J. Watkins \& O. C. Watkins, 1976), recall is mediated by retrieval cues, and these cues are subject to overload. Cue effectiveness varies inversely with the number of episodes or propositions that the cue subsumes. In Experiment 1 , the central fact was used as the cue. In the single condition, the fact cue subsumed only one proposition, the name of the historical figure. In the related condition the fact cue subsumed not only the name of the historical figure but also the two related facts. The recall differences between the two conditions therefore could be explained by cue overload.

The purpose of Experiment 2 was to determine whether the results found in Experiment 1 were entirely due to cue overload. To do this, a second cue condition was added. In the new cue condition, subjects were presented with all of the facts (either one or three) that had been presented with the historical figures. With the addition of this type of cue (multiple-fact), cue overload could be assessed as an explanation of any observed recall differences.

\section{EXPERIMENT 2}

Experiment 2 was a reproduction of Experiment 1, except that a second type of recall test was included. In this experiment, half of the subjects were cued with all the facts that had been presented with the historical figures. This was done to determine whether the recall differences found in Experiment 1 were attributable to cue overload.

\section{Method}

The method of Experiment 2 was identical to that used in Exyeriment 1 except for the differences noted below.

Subjects. Subjects were 84 undergraduate students at the Univerity of New Hampshire. The students received course credit for heir participation.

Procedure. Experiment 2 again took place in three different sesions in which stimulus items in one of the three conditions were resented. In each session, half of the subjects were given only the central facts as cues, as was done in Experiment 1 . The other half of the subjects were given all the facts (one or three) that had been presented in relation to a historical figure. The multiple facts for each historical figure were presented in a group so that it was discernible that the three facts all referred to a single figure.

\section{Results and Discussion}

Table 2 shows the mean percentage of correctly recalled names for condition and test type. A fact condition (single, related, unrelated) $\times$ test type (central-fact cue, multiple-fact cue) analysis of variance on the number of names recalled yielded a significant main effect of fact condition $(F=9.02, M S e=1.60, p<.001)$. A Tukey comparison test revealed that the single-fact condition yielded better name recall than did the related- or unrelated-fact conditions. Neither the effect of test type nor the interaction was significant. However, the differences between the three conditions did lessen in the multiple-fact cue condition. The results of this experiment clearly do not support the predictions of J. R. Anderson's (1983) model and weaken the argument that the results of Experiment 1 were due to cue overload.

The results of the first two experiments provide strong evidence that the relative effects of providing additional sources of activation and redirecting activation play, at best, a very small role in increasing recall performance. In fact, the results of these two experiments seem to suggest that when the role of reconstructive processes are eliminated, the amount of elaboration is inversely related to recall performance. If this is true, recall performance should decrease as the number of associated pathways connecting the name to the facts increases.

The design of the next two experiments was also changed in an attempt to control for initial learning. It could be that the recall differences found in the first two experiments were due to differences in the amounts of initial learning of the single and related sentences, attributable to the fact that more time per sentence was allotted for the single condition. Two different trials-tocriterion procedures were used in Experiments 3 and 4 , and a self-paced learning procedure was used in Experiment 4 in an effort to ensure that all facts were stored in long-term memory.

\section{EXPERIMENT 3}

In this experiment, the number of facts presented that related to the historical figure was systematically varied. In addition, a trials-to-criterion procedure was used to try to equate for initial learning. These modifications permitted a test of the inverse relationship between amount of elaboration and recall performance.

\section{Method}

Subjects. Subjects were 40 undergraduate students at the University of New Hampshire. The students received course credit for their participation.

Materials. The same basic stimuli were used in this experiment as were used in the earlier experiments except for the addition of 
new facts. First, a new related fact was found for each of the 21 central facts. Second, 3 new historical figures, with their associated central facts and 3 related facts each, were added to the stimulus list. The addition of these new facts meant that there were 24 central facts relating to historical figures. Twelve of the historical figures had 3 related facts that were probable outcomes, and 12 had 3 related facts that were probable causes of the central fact.

Subjects read all 24 central facts. The number of additional facts associated with each central fact was counterbalanced across subjects so that each central fact was read by an equal number of subjects in each of the four fact conditions (single-fact, two-facts, threefacts, four-facts).

Procedure. The procedures used in this experiment were the same as those used in Experiment 1, except for the following changes. First, subjects were paced through the memorization phase of the study. Subjects were given $15 \mathrm{sec}$ for single facts, $20 \mathrm{sec}$ for two facts, $30 \mathrm{sec}$ for three facts, and $40 \mathrm{sec}$ for four facts. Following completion of the memorization phase, subjects were given the central and related facts for each historical figure. The subjects were asked to write the name associated with each fact set. Responses were scored and the correct name provided for each error. Subjects were then given a new sheet of facts and asked to provide the name for each set of facts that had been incorrectly answered on the first trial. Three different orders of the fact sheets were used to ensure that the students could not remember the correct name based on order or location. This procedure of study followed by test was continued until each subject had correctly identified each set of facts. As soon as all subjects had reached criterion, they spent 4 min working on a 10-item number-series task. Following this distractor task, subjects were given a booklet containing the 24 central facts and asked to write down the names of the appropriate historical figures.

\section{Results and Discussion}

A one-way analysis of variance on the number of correctly recalled historical figures failed to show a significant effect of number of related facts $(F=1.76$, $p<.20$ ). A trend analysis, however, did reveal a significant linear trend $(F=6.63, p<.02)$. The mean number of recalled names was $4.15,3.95,3.88$, and 3.60 for the single-fact, two-fact, three-fact, and four-fact conditions, respectively. The respective standard deviations were $1.27,1.09,1.20$, and 1.30 . The finding of a significant linear trend supports the position that there is an inverse relationship between the amount of elaboration and recall when the role of inferential reconstruction has been eliminated.

Although the analysis of linear trends found the predicted inverse relationship between name recall and amount of elaboration, it is possible that this result is due to differences among fact conditions in terms of the number of practice trials. If the conditions differed on number of study trials, the recall differences could be due to differences in the amount of delay between studying and testing.

To check for this possibility, an analysis of variance was performed on the mean number of trials-to-criterion for the items in each fact condition. This analysis failed to reveal any differences due to fact condition. The mean number of trials per condition was 2.41 for the singlefact, 2.37 for the two-facts, 2.32 for the three-facts, and 2.51 for the four-facts condition.

\section{EXPERIMENT 4}

The purpose of the final experiment was to test once again the relative effects of elaboration but with a new initial learning procedure. In this experiment, both an overlearning procedure and a self-paced study procedure were used to try to reduce the possibility of differences in initial learning between the elaborated and single-fact conditions. In addition, these new procedures allowed a closer scrutiny of possible differences in the amount of study time needed for the two conditions and the relative ease of acquisition.

\section{Method}

Subjects. Subjects were 28 undergraduate students enrolled at the University of New Hampshire. Subjects were recruited from several psychology classes and paid $\$ 5$ for their participation in the study.

Materials. A subset of the stimuli used in the earlier experiments was also used in Experiment 4. Sixteen historical figures and the associated central facts were used with two related sentences. Each subject in the experiment was presented with information about 8 historical figures in the single-fact condition and 8 in the elaborated (three related sentences) condition. As before, central facts were presented to an equal number of students in each of the conditions.

Procedure. Subjects in this experiment participated in two experimental sessions. The first session was the learning phase, and the second session, which occurred 1 week later, was the test phase. Subjects were run individually during the first session.

In the learning phase, each student was told that he/she would be asked to learn information concerning 16 famous historical figures. They were told that the purpose of the experiment was twofold. One purpose was to discover how subjects would allocate their study time for each of the stimuli. The second purpose was to discover what relationship existed between the amount of study time and recall of the information. As in the earlier studies it was stressed that both the names and the associated information must be learned. In addition, students were told that each of 8 historical figures would have one fact associated with him/her, whereas each of the other 8 figures would have three facts associated with him/her. Examples were given of each type.

Each student was informed that the first experimental session would consist of at least four study-test trials. In the first trial, the stimuli were presented one at a time via the video monitor of a Radio Shack TRS- 80 computer. The student controlled the length of study time for each item by pressing any key on the computer keyboard. When a key was pressed, the current item was replaced by the next stimulus, and the amount of study time was recorded by the computer. In the elaborated condition, all three facts were presented on the screen simultaneously, with the central fact presented at the top of the screen. It was stressed that each item should be studied until the student felt that it could later be recalled. After studying all 16 items, the student was given a test booklet that consisted of all of the test items, with the names of the historical figures removed. The student was instructed to provide the name for each set of facts. Once the test was completed, the experimenter scored the tests, pointed out the student's errors, and then began the second trial. In the second and all subsequent trials, each student studied from a booklet that contained all the historical figures and the associated facts and his/her corrected exam from the previous trial. Again study time was determined by each student, but the students could study items in any order and could go back and restudy earlier items. Students continued to study until they felt that 
they had mastered the material and requested another exam. This series of study-test trials continued until each student had four consecutive trials in which all the names were provided for the 16 sets of facts. In all trials, both the central fact and the related facts (for the elaborated condition) were presented together. Three different random orders were used for the initial tests.

Once students reached the criterion of four successive perfect trials, they were informed that the data obtained regarding study time and recall performance would be used to design a future test of their study habits. They were given an appointment for a second experimental session the following week (a 7-day delay). At the second session, all students were given a new random order of the test used in the first experimental session. Their recall performance was the dependent measure of primary interest.

\section{Results and Discussion}

Initially, analyses of variance were performed to discover whether differences existed between the two fact conditions in amount of initial study time and ease of learning. The first analysis revealed a significant difference in average study time (in the first trial) between the elaborated condition (mean $=29.451 \mathrm{msec}$ ) and the single-fact condition (mean $=14.832 \mathrm{msec}$ ). Analyses performed on the number of items recalled during the first trial and on the number of trials to reach the criterion of four perfect recalls yielded no difference due to fact condition. Overall, subjects required an average of 5.92 trials to reach criterion.

The comparison of primary interest was made by performing an analysis of variance on the mean number of correctly recalled names for the delayed test. This analysis revealed a significant difference $(F=4.37$, $M S \mathrm{e}=0.73, p<.05)$. An average of $6.02(75.3 \%)$ of the names were recalled in the single-fact condition and an average of $5.48(68.5 \%)$ of the names were recalled in the elaborated condition. Thus, once again, names were better recalled when they had been stored in a less elaborated memory trace.

\section{GENERAL DISCUSSION}

The purpose of the experiments reported in this paper was to provide an initial test of the effectiveness of elaborative processing in increasing recall performance through direct retrieval. In all four experiments, recall performance was found to decrease with an increased amount of elaboration in the memory trace. Overall, then, the results provide strong counterevidence to J. R. Anderson's (1983) claim that elaborative processing increases recall through direct retrieval. These results suggest that the beneficial effect of elaboration on recall performance may be due solely to inferential reconstruction, with the role of additional sources of activation and redirecting activation being negligible, at best.

All four experiments reported here clearly showed that recall of a name was better when there were fewer connections to other propositions. This finding may at first seem to contradict research that shows that the amount of integration among propositions determines recall performance (e.g., Hays-Roth \& Thorndyke, 1979; Hupet
\& LeBouedec, 1977). However, studies of this type may not have controlled for the inferential redundancy across propositions. It seems that if one cannot use associated information to reconstruct a proposition, elaboration serves only to decrease the probability of recall of a single proposition. Such an explanation would also encompass both these results and those of earlier work on elaboration (e.g., Bradshaw \& J. R. Anderson, 1982).

Although the experiments reported in this paper find no evidence for increased recall performance with increased elaboration of a trace, J. R. Anderson's (1983) theoretical formulation still seems sound. Evidence of the beneficial effects of multiple pathways available for retrieval is not disputed. However, the structure of a memory trace that allows these processes to operate also has its costs. One obvious cost, as J. R. Anderson noted, is that the amount of activation that spreads from an activated proposition to another proposition in the trace depends, in part, upon the total number of pathways leading from the activated proposition.

Elaborative processing, by definition, serves to increase the number of pathways, thereby decreasing the amount of activation that flows directly from the activated proposition to the target proposition. For elaborative processing to increase recall performance through direct retrieval, therefore, the benefits of having alternative retrieval pathways must outweigh the cost of less direct activation. In a test of speed of retrieval, elaborative processing hinders performance. However, in an untimed recall task, how can the provision of additional retrieval pathways hurt performance? Based upon previous work, there seem to be two ways this can happen. First, when encoding new information into memory, one may not increase the amount of processing in direct proportion to the number of pathways or propositions in the trace. This would lead to a decrease in the amount of processing that any one pathway in an elaborated trace would receive. This could especially happen if the person employs a guessing strategy, where a guess is made regarding which of the facts is most important or easiest to learn, and then the person focuses most of his/her processing on that fact during study. If this is the case, the relative strength of the associative pathways may differ between information stored alone or in a more elaborated trace. For example, there is a large body of literature (e.g., Johnson-Laird \& Bethell-Fox, 1978; Walker, Jones, \& Mar, 1983) that suggests that the amount of processing involved in creating or rehearsing a pathway between two propositions increases the relative strength of the pathway. As the strength of the pathway increases, the amount of activation energy needed to spread down the pathway decreases, making the pathways more accessible at time of retrieval. It may be that the amount of processing energy used to create each pathway in an elaborated trace is less than the amount of processing expended in creating the one or two pathways in the less elaborated trace. Therefore, the relative strength of any one pathway would be greater in the less elaborated trace. If this occurs, there will be some situations 
in which the amount of activation that spreads from the cue proposition will not be sufficient to activate any of the propositions in the elaborated trace. In the less elaborated trace, on the other hand, with the greater associative strength of its fewer pathways, the amount of activation may be sufficient to spread to the to-be-recalled proposition. This explanation suggests that the relative effectiveness of elaboration, especially when inferential reconstruction is not possible, would be dependent upon the level of accessibility of the information at time of retrieval, as suggested by Yekovich and Manelis (1980).

The second way in which provision of alternative pathways could reduce recall in practice is through the failure of a person to perform an exhaustive, or at least sufficient, search. In the procedures used in these experiments, recall of a name in the single condition depends on the amount of activation that spreads from the cue to the name. This is an automatic process, independent of a person's use of a strategy. Little or no active processing is required. However, for refocusing to occur, a person must actively process the information by retrieving the activated proposition contained in the elaborated information, refocusing upon it, and then continuing this chain of processes until the to-be-recalled proposition has been activated. This process, because it is under the control of the person, may be terminated before the target proposition has been located. Terminating the process may be due to a strategy bias of the individual or to lack of incentive to continue the search (a likely possibility in many memory experiments).

Overall, this formulation does not suggest that elaborative processing cannot improve recall through direct retrieval. The presence of multiple retrieval pathways increases the probability that some link between a cue and the to-be-recalled information still exists (this is the concept of network redundancy proposed by Bradshaw and J. R. Anderson, 1982). Therefore, there could be advantages to a more elaborated memory trace, depending upon the probability of a loss of one or more of the pathways. However, the assumption that recall performance is always improved by increased amounts of network redundancy in the memory trace is incorrect. The data presented in this paper demonstrate that, under at least some conditions, recall performance is inversely related to the amount of elaboration in the memory trace.

\section{REFERENCES}

Anderson, J. R. (1983). The architecture of cognition. Cambridge, MA: Harvard University Press.

ANDERson, J. R., Bower, G. H. (1972). Configural properties in sentence memory. Journal of Verbal Learning \& Verbal Behavior, 11, 594-605.

ANDERSON, J. R., \& REDER, L. M. (1979). An elaborative processing explanation of depth of processing. In L. Cermak \& F. Craik (Eds.), Levels of processing in human memory. Hillsdale, NJ: Erlbaum.

Anderson, R. C., ORTONY, A. (1975). On putting apples into bottles-a problem of polysemy. Cognitive Psychology, 7, 167-180.

BRADSHAW, G. L., \& ANDERSON, J. R. (1982). Elaborative encoding as an explanation of levels of processing. Journal of Verbal Learning \& Verbal Behavior, 21, 165-174.

EYSENCK, M. W. (1979). Depth, elaboration, and distinctiveness. In L. Cermak \& F. Craik (Eds.), Levels of processing in human memory. Hillsdale, NJ: Erlbaum.

HAYES-Roth, B., \& THORNDYKe, P. W. (1979). Integration of knowledge from test. Journal of Verbal Learning \& Verbal Behavior, 18, 91-108.

HuPET, M., \& LeBouedec, B. (1977). The given-new contract and the constructive aspect of memory for ideas. Journal of Verbal Learning \& Verbal Behavior, 16, 69-75.

Johnson-Laird, P. M., \& Bethell-Fox, C. E. (1978). Memory for questions and the amount of processing. Memory \& Cognition, 6 , 496-501.

MUElleR, C. W., \& WATKINS, M. J. (1977). Inhibition from part-set cuing: A cue-overload interpretation. Journal of Verbal Learning \& Verbal Behavior, 16, 699-709.

Stein, B. S., Littlefield, J., Bransford, J. D., \& Persampieri, M. (1984). Elaboration and knowledge acquisition. Memory \& Cognition, 12, 522-529.

Stein, B. S., Morris, C. D., \& Bransford, J. D. (1978). Constraints on effective elaboration. Journal of Verbal Learning \& Verbal Behavior, 17, 707-714.

VAN DuJK, T. A., KINTSCH, W. (1983). Strategies of discourse comprehension. New York: Academic Press.

WALKER, N., JoNES, J. P., \& MAR, H. H. (1983). Encoding processes and the recall of text. Memory \& Cognition, 11, 275-282.

Watkins, M. J., \& Watkins, O. C. (1976). Cue-overload theory and the method of interpolated attributes. Bulletin of the Psychonomic Society, 7, 289-291.

Yekovich, F. R., \& Manelis, L. (1980). Accessing integrated and nonintegrated propositional structures in memory. Memory \& Cognition, 8, 133-140. 